\title{
Intrinsic curvature of thermodynamic potentials for black holes with critical points
}

\author{
Brian P. Dolan* \\ Department of Mathematical Physics, Maynooth University, Maynooth, Ireland \\ and Dublin Institute for Advanced Studies, 10 Burlington Road, Dublin, Ireland
} (Received 22 April 2015; published 11 August 2015)

\begin{abstract}
The geometry of thermodynamic state space is studied for asymptotically anti-de Sitter black holes in $D$-dimensional space-times. Convexity of thermodynamic potentials and the analytic structure of the response functions is analyzed. The thermodynamic potentials can be used to define a metric on the space of thermodynamic variables, and two commonly used such metrics are the Weinhold metric, derived from the internal energy, and the Ruppeiner metric, derived from the entropy. The intrinsic curvature of these metrics is calculated for charged and for rotating black holes, and it is shown that the curvature diverges when heat capacities diverge but, contrary to general expectations, the singularities in the Ricci scalars do not reflect the critical behavior. When a cosmological constant is included as a state space variable, it can be interpreted as a pressure and the thermodynamically conjugate variable as a thermodynamic volume. The geometry of the resulting extended thermodynamic state space is also studied, in the context of rotating black holes, and there are curvature singularities when the heat capacity at constant angular velocity diverges and when the black hole is incompressible. Again the critical behavior is not visible in the singularities of the thermodynamic Ricci scalar.
\end{abstract}

DOI: 10.1103/PhysRevD.92.044013

PACS numbers: 04.50.Gh, 04.70.Dy, 05.70.-a

\section{INTRODUCTION}

Thermodynamics is a very general yet remarkably powerful tool for understanding the physical properties of a very wide range of phenomena. Its generality lies in the fact that the formalism sidesteps direct questions about the nature of the underlying microscopic physics. This is an obvious advantage when studying systems whose microscopic physics is not yet well understood, such as the physics of quantum gravity. The quantum nature of black holes is thus a perfect arena for thermodynamics to be used as an early stage investigative tool for gaining insight into the underlying microscopic physics.

The aim of the present study is to elucidate the geometry of black hole thermodynamic potentials. For a gas thermodynamic stability demands that the thermal energy be a convex function of the entropy $S$ and the volume $V$, from which the well known relation $C_{P}>C_{V}$ follows. The isothermal compressibility $\kappa_{T}$ and the isentropic compressibility $\kappa_{S}$ are related to the heat capacities by $C_{P} \kappa_{S}=C_{V} \kappa_{T}$, implying the reciprocal relation $\kappa_{T}>\kappa_{S}$. Schwarzschild black holes famously have negative heat capacity [1] but stability can be achieved in asymptotically anti-de Sitter space, with a suitable cosmological constant [2]. For a black hole, with electric charge $Q$, we replace $V$ with $Q$ and the analogous condition for the heat capacities ${ }^{1}$ is then

\footnotetext{
*bdolan@thphys.nuim.ie

${ }^{1}$ Strictly speaking it is the enthalpy of the black hole that is the relevant thermodynamic potential when a fixed negative cosmological is present, rather than the internal energy [3]. At fixed pressure the enthalpy should be a convex function of $S$ and $Q$.
}

$C_{\Phi}>C_{Q}$, where $\Phi$ is the electrostatic potential of the black hole. In this case the isothermal electrical capacitance, $\mathcal{C}_{T}=\left.\frac{\partial Q}{\partial \Phi}\right|_{T}$, and the isentropic electrical capacitance, $\mathcal{C}_{T}=\left.\frac{\partial Q}{\partial \Phi}\right|_{S}$, are related to the heat capacities by $C_{\Phi} \mathcal{C}_{S}=C_{Q} \mathcal{C}_{T}$, implying $\mathcal{C}_{T}>\mathcal{C}_{S}$. For rotating black holes in asymptotically anti-de Sitter (AdS) space-time $V$ is replaced with the angular momentum, $J$, and $-P$ by the appropriate angular velocity, $\Omega$. The analogue of the compressibilities for a gas are the moments of inertia, $\mathcal{I}_{S}=$ $\left.\frac{\partial J}{\partial \Omega}\right|_{S}$ and $\mathcal{I}_{T}=\left.\frac{\partial J}{\partial \Omega}\right|_{T}$. For stability $C_{\Omega}>C_{J}$ and $C_{\Omega} \mathcal{I}_{S}=$ $C_{J} \mathcal{I}_{T}$ implies $\mathcal{I}_{T}>\mathcal{I}_{S}$. The analytic structure of the response functions, in particular the interplay between their zeros and singularities, is crucial in satisfying these relations, and we shall explore this in detail for charged and rotating black holes in space-time dimensions $D \geq 4$. For example, the phenomenon of ultraspinning black holes in $D>4$ is associated with a negative moment of inertia.

Thermodynamic potentials can also be used to generate a metric on the space of thermodynamic states so that the intrinsic curvature associated with the metric encodes information about the underlying physics of the thermodynamic system [4,5] (for a review see [6]). The study of the geometry of thermodynamic state space for black holes was pioneered in [7] and has subsequently been investigated by a number of authors (a nonexhaustive list is provided by [8-24], and there is a review in [25]). In the present work this program is taken further and curvatures are studied for charged and for rotating black holes in $D$-dimensional space-times for all $D \geq 4$, in the presence of a cosmological constant $\Lambda$. Generically one expects the 
intrinsic curvature of thermodynamic state space to diverge when response functions, such as heat capacities, diverge $[6,26]$, and this expectation is indeed realized, but we shall see that there is a twist for black holes in that critical points, which are known to exist for $\Lambda<0$, are not visible in the thermodynamic curvatures calculated here.

When the cosmological constant is included in the set of thermodynamic variables, as argued in [3] it should be, the dimension of the thermodynamic state space is increased. ${ }^{2}$ Most of the literature to date on the thermodynamic geometry of black holes has assumed fixed $\Lambda$, but when state space is extended to include $\Lambda$ the dimension of the thermodynamic space is increased and the calculations become more involved. The thermodynamic geometry of black holes with varying $\Lambda$ was considered in [33-35] but the study in the present paper is rather different in that it also considers the role of the thermodynamic volume, the Legendre transform of the pressure, which to our knowledge has so far not been considered as a state variable in the thermodynamic geometry of black holes.

The layout of the paper is as follows. In Sec. II the geometry of thermodynamic state space is briefly reviewed; Sec. III concerns charged, nonrotating black holes in $D$ space-time dimensions and extends known results in $D=4$ for spherical black holes to $D>4$ and general event horizon topologies; Sec. IV discusses the thermodynamic geometry of neutral singly spinning black holes in $D$-dimensions, the curvature scalar associated with the thermodynamic geometry is discussed, and singularities in the curvature scalar are related to singularities in the heat capacity and vanishing compressibility. Finally Sec. V summarizes the conclusions while some technical results are given in two appendixes.

\section{THERMODYNAMIC METRICS}

Different thermodynamic potentials give rise to different thermodynamic metrics, and both the internal energy, $U(S, V)$, and the entropy, $S(U, V)$, can be used to generate Euclidean signature metrics for thermodynamically stable systems [6]. For example, the internal energy of a single component gas is a convex function of the extensive variables, entropy $S$ and volume $V$, and the Hessian matrix

$$
g_{A B}=\frac{\partial^{2} U}{\partial X^{A} \partial X^{B}},
$$

with $\left(X^{1}, X^{2}\right)=(S, V)$ and $A, B=1,2$, is a positive definite matrix. Viewed as a metric, originally considered by Weinhold [4], there is an intrinsic curvature associated

\footnotetext{
${ }^{2}$ The possibility of varying $\Lambda$ was first considered by Henneaux and Teitelboim [27] and has been revisited by various authors since [28-32]. Although the focus here is on negative $\Lambda$, many of the formulas can be analytically continued to positive $\Lambda$.
}

with this matrix which has been calculated for a number of thermodynamic systems [6].

The components of $g_{A B}$ are related to the response functions of the thermodynamic system,

$$
g_{S S}=\left.\frac{\partial T}{\partial S}\right|_{V}=\frac{T}{C_{V}}
$$

with $C_{V}$ the heat capacity at constant volume;

$$
g_{V V}=-\left.\frac{\partial P}{\partial V}\right|_{S}=\frac{1}{V \kappa_{S}}
$$

where $\kappa_{S}=-\left.\frac{1}{V} \frac{\partial V}{\partial P}\right|_{S}$ is the adiabatic compressibility, and

$$
g_{S V}=\frac{1}{V \alpha_{S}},
$$

where $\alpha_{S}=\left.\frac{1}{V} \frac{\partial V}{\partial T}\right|_{S}$ is the adiabatic thermal expansion coefficient. Standard thermodynamic relations can be used ${ }^{3}$ to show that

$$
\operatorname{det} g=\frac{T}{V C_{P} \kappa_{S}} .
$$

A generalization of this formula when there are more than two independent thermodynamic variables was given in [26].

When response functions diverge, the determinant of the metric vanishes and it will not be invertible. In a thermodynamic system with two variables the locus of points on which a response function diverges is called a spinodal curve, and it is natural to ask whether this lack of invertibility on a spinodal curve is associated with a genuine singularity in the intrinsic curvature or if it is just a coordinate singularity. Indeed the thermodynamic curvature is inversely proportional $(\operatorname{det} g)^{2}$ and is expected to diverge on spinodal curves $[6,26]$. For the van der Waals gas for example the curvature is proportional to $\left(C_{P} \kappa_{S}\right)^{2}$ and diverges when $C_{P}$ diverges. The spinodal curve has two branches in the $P-V$ plane, and with the exception of the critical point where the two branches meet, it lies in a regime that is not thermodynamically stable.

A related metric, the Ruppeiner metric, is associated with the Hessian matrix of the entropy, $S(U, V)$, which is a concave function, so

\footnotetext{
${ }^{3}$ In particular, the aforementioned relation $C_{P} \kappa_{S}=C_{V} \kappa_{T}$ together with $C_{P}=C_{V}+\frac{T V \alpha_{P}^{2}}{\kappa_{T}}$ and $\kappa_{T}=\kappa_{S}+\frac{T V \alpha_{P}^{2}}{C_{P}}$, where $\kappa_{T}=$ $-\left.\frac{1}{V} \frac{\partial V}{\partial P}\right|_{T}$ is the isothermal compressibility, and $\alpha_{P}=\left.\frac{1}{V} \frac{\partial V}{\partial T}\right|_{P}$ is the isobaric thermal expansion coefficient. Also the Maxwell relation $\left.\frac{\partial T}{\partial V}\right|_{S}=-\left.\frac{\partial P}{\partial S}\right|_{V}$ implies

$$
g_{S V}=-\left.\left.\frac{\partial P}{\partial T}\right|_{V} \frac{\partial T}{\partial S}\right|_{V}=\left.\left(\left.\left.\frac{\partial V}{\partial T}\right|_{P} \frac{\partial P}{\partial V}\right|_{T}\right) \frac{\partial T}{\partial S}\right|_{V}=-\frac{\alpha_{P}}{\kappa_{T}} \frac{T}{C_{V}}
$$
}




$$
\tilde{g}_{A B}=-\frac{\partial^{2} S}{\partial \tilde{X}^{A} \partial \tilde{X}^{B}},
$$

with $\left(\tilde{X}^{1}, \tilde{X}^{2}\right)=(U, V)$, is also a positive definite matrix if the system is stable. It has been argued [6] that the intrinsic thermodynamic curvature contains information about microscopic physics of the system; for example, it diverges when response functions diverge. Indeed both curvatures vanish for an ideal gas and both are positive for a van der Waals gas and diverge at the critical point.

The Ruppeiner and the Weinhold metrics are conformally related,

$$
\tilde{g}_{A B}=\frac{1}{T} g_{A B}
$$

and give equivalent information. The Ricci scalar of the Weinhold metric, $R$, and that of the Ruppeiner metric $\tilde{R}$, are therefore related by the standard formula for conformally related metrics,

$$
\tilde{R}=T R+(n-1) \nabla^{2} T-\frac{(n-1)(n+2)}{4} \frac{(\nabla T)^{2}}{T},
$$

where $n$ is the number of independent thermodynamic variables, ${ }^{4}$ one of which is $T$. The second term on the righthand side involves

$$
\begin{aligned}
\nabla^{2} T & =\frac{1}{\sqrt{\operatorname{det} g}} \partial_{a}\left(\sqrt{\operatorname{det} g} g^{a b} \partial_{b} T\right) \\
& =\frac{1}{\sqrt{\operatorname{det} g}} \partial_{a}\left(\sqrt{\operatorname{det} g} g^{a b} g_{b S}\right) \\
& =\left.\frac{1}{\sqrt{\operatorname{det} g}} \frac{\partial \sqrt{\operatorname{det} g}}{\partial S}\right|_{V} .
\end{aligned}
$$

The last term on the right-hand side of (5) is related to the heat capacity at constant volume, $C_{V}=\frac{T}{g_{S S}}$,

$$
\frac{(\nabla T)^{2}}{T}=\frac{1}{C_{V}}
$$

since

$$
\nabla_{a} T=\partial_{a} T=g_{a S} \Rightarrow(\nabla T)^{2}=g^{a b} g_{a S} g_{b S}=g_{S S}=\frac{T}{C_{V}}
$$

from (2). When $n=2$ and the only variables are $(S, V)$ the relation between the Weinhold and the Ruppeiner curvatures is

\footnotetext{
${ }^{4}$ When the variables are $(S, V)$, then $n=2$, but we quote the more general result.
}

$$
\tilde{R}=T R-\frac{1}{2} \partial_{S} \ln \left(\frac{T C_{V} \kappa_{T}}{V}\right)
$$

(the derivative here is at constant $V$ and the factor of $V$ has been chosen to make the argument of the logarithm dimensionless).

Another class of conformally related metric was proposed in [36], but the focus here will be restricted to the specific cases (1) and (4); otherwise the analysis would become rather too long.

The thermodynamic potentials for a gas also depend on the number of particles so, for a single component gas consisting of $N$ particles, $U(S, V, N)$, and a complete description requires three independent variables, $X^{A}=$ $(S, V, N)$ with $A=1,2,3$. One might expect that a full description of the thermodynamic geometry would require calculating a $3 \times 3$ Ricci tensor, but this is not the case in standard thermodynamics as the resulting $3 \times 3$ Hessian matrix is not invertible and cannot be used to determine a curvature. This is a consequence of the Gibbs-Duhem relation, which follows from homogeneous scaling of the extensive thermodynamic state variables and potentials. Consider the Gibbs free energy,

$$
\mathcal{G}(T, P, N)=\mu N=U(S, V, N)-T S+P V,
$$

where $\mu=\left.\frac{\partial U}{\partial N}\right|_{S, V}$ is the chemical potential. In the threedimensional state space parametrized by extensive variables $X^{A}=(S, V, N)$ this can be written as

$$
U(X)=X^{A} Y_{A}(X),
$$

where $Y_{A}(X):=\partial_{A} U$. Now the $3 \times 3$ Hessian matrix is

$$
g_{A B}=\partial_{A} Y_{B}
$$

so

$$
Y_{A}=\partial_{A} U=Y_{A}+g_{A B} X^{B},
$$

where (9) has been used for the second equality. Hence $g_{A B} X^{B}=0$ and the vector $\vec{X}=\left(X^{1}, X^{2}, X^{3}\right)$ is an eigenvector of $g_{A B}$ with eigenvalue zero (this is essentially a consequence of homogeneous scaling). Assuming the other two eigenvalues are finite, the $3 \times 3$ Hessian matrix is never invertible and is not a suitable candidate for a positive definite metric. Similarly for a multicomponent gas, with $k$ different chemical species $i=1, \ldots, k$ and particle numbers $N_{i}$, there is always at least one zero eigenvector of the $(k+2) \times(k+2)$ Hessian matrix. For this reason it is natural to fix the total particle number $N=N_{1}+\cdots N_{k}$ and only consider a $(k+1) \times(k+1)$ metric.

For black holes we do not yet have an analogue of the particle number; nevertheless scaling arguments can be applied to derive the black hole analogue of the GibbsDuhem relation, the Smarr relation [37]. For black holes the 
extensive thermodynamic variables do not scale homogeneously. For example, in $D$ space-time dimensions the black hole mass, $M$, and electric charge, $Q$, have dimension $(D-3)$ (in units with $G=c=1$ ), while entropy, $S$, and angular momentum, $J$, have dimension $(D-2)$ and $\Lambda$ has dimension -2 . If $\Lambda$ is equated to a pressure,

$$
\Lambda=-8 \pi P,
$$

so negative $\Lambda$ gives a positive pressure. Then the black hole mass has the thermodynamic interpretation of enthalpy [3].

The mass is a function of entropy, angular momentum $J^{i}$ [where $i=1, \ldots, r$ and $r$ is the rank of $S O(D-1)$ ], electric charge, and pressure: $M\left(S, J^{i}, Q, P\right)$. The thermodynamically conjugate variables are temperature, angular velocity $\Omega_{i}$, electric potential $\Phi$, and thermodynamic volume $V$,

$$
\begin{gathered}
T=\frac{\partial M}{\partial S}, \quad \Omega_{i}=\frac{\partial M}{\partial J^{i}}, \\
\Phi=\frac{\partial M}{\partial Q}, \quad \text { and } \quad V=\frac{\partial M}{\partial P} .
\end{gathered}
$$

The Smarr relation, the black hole analogue of (8), then reads

$$
\begin{aligned}
(D-3) M= & (D-2) \Omega . \mathbf{J}+(D-2) T S-2 P V \\
& +(D-3) Q \Phi .
\end{aligned}
$$

When $\Lambda$ is allowed to vary, the Weinhold metric should be defined in terms of the internal energy $U$ in order to have a positive definite metric. The internal energy is the Legendre transform of the enthalpy with respect to $P$,

$$
U\left(S, J^{i}, Q, V\right)=M-P V .
$$

Then

$$
T=\frac{\partial U}{\partial S}, \quad \Omega_{i}=\frac{\partial U}{\partial J^{i}}, \quad \Phi=\frac{\partial U}{\partial Q}, \quad P=-\frac{\partial U}{\partial V},
$$

and

$$
g_{A B}=\frac{\partial^{2} U}{\partial X^{A} \partial X^{B}},
$$

with $X^{A}=\left(S, J^{i}, Q, V\right)$ and $A=1, \ldots, r+3$.

One immediate consequence of the inhomogeneous scaling of black hole thermodynamic functions is that, unlike ordinary thermodynamics, $g_{A B}$ need not have a zero eigenvalue. However, if $P=0$, it always has a negative eigenvalue [38], and there are no thermodynamically stable asymptotically flat black holes in any dimension, regardless of rotation or charge. On the other hand, asymptotically AdS black holes can be stabilized by a large enough positive pressure: Hawking and Page realized this for nonrotating neutral black holes [2], and it was generalized to the charged nonrotating case in [39] and the charged rotating case in [38].

Note that the complete Legendre transform of $U$,

$$
\mathcal{E}(\Omega, T, P, \Phi):=U-\Omega_{i} \mathbf{J}^{i}-T S+P V-\Phi Q,
$$

is nonzero for black holes - in contrast to ordinary thermodynamics where (8), and its generalization to multicomponent systems, implies that

$$
U-T S+P V-\mu_{i} N^{i}=0 .
$$

This is again a consequence of the inhomogeneous scaling of black hole thermodynamic variables and $\mathcal{E}$ is in fact related to the Euclidean action, $I_{E}$, of the black hole via [40]

$$
\mathcal{E}=T I_{E}
$$

When discussing the thermodynamic geometry of asymptotically AdS black holes, it is very common in the literature to use the enthalpy $M\left(S, J^{i}, Q, P\right)$ rather than the internal energy $U\left(S, J^{i}, Q, V\right)$ to derive a Weinhold-like metric. This not unreasonable when $P$ is fixed, and indeed for asymptotically flat black holes with $P=0$ it makes no difference which one uses as they are the same. When $P \neq 0$, the $(r+2)$-dimensional metric obtained from the Hessian of $M$ by varying $S, Q$, and $J^{i}$ with $P$ fixed will be positive definite for a thermodynamically stable black hole. Typically there are curvature singularities when the heat capacity diverges.

In [33] $P$ was varied along with the other parameters but this has the disadvantage that the Hessian matrix of $M\left(S, J^{i}, Q, P\right)$ cannot be expected to be positive definite for thermodynamically stable systems; one should use $U\left(S, J^{i}, Q, V\right)$ to get a positive definite Weinhold metric.

A technical point is that, for a given solution of Einstein's equations, an explicit expression for the mass in terms of thermodynamic variables is often cumbersome at best and intractable at worst. It is usually more convenient to express the thermodynamic potentials in terms of variables other than $\left(S, J^{i}, Q, V\right)$. Curvature tensors can of course be calculated in any coordinate system, invoking general coordinate covariance, but one must be careful because the right-hand side of (11) is not covariant under general coordinate transformations: $X^{A}=$ $\left(S, J^{i}, Q, V\right)$ are a privileged set of coordinates that picks out a particular Lagrangian submanifold of thermodynamic "phase space" [36]. Writing the thermodynamic line element as

$$
d^{2} s=\frac{\partial^{2} U}{\partial X^{A} \partial X^{B}} d X^{A} d X^{B}=d Y_{A} d X^{A},
$$

where $Y_{A}=\left(T, \Omega_{i}, \Phi,-P\right)$, we can change variables from $X^{A}$ to $X^{A^{\prime}}(X)$ in terms of which Eq. (13) becomes 


$$
d^{2} s=g_{A^{\prime} B^{\prime}} d X^{A^{\prime}} d X^{B^{\prime}}
$$

with

$$
g_{A^{\prime} B^{\prime}}=\frac{\partial Y_{C}}{\partial X^{A^{\prime}}} \frac{\partial X^{C}}{\partial X^{B^{\prime}}}
$$

The metric thus factorizes into a product of two matrices. Equation (14) will prove to be the most practical starting point for calculating curvatures in an arbitrary coordinate system $X^{A^{\prime}}$ for the thermodynamic state space when the functional forms of $Y_{A}\left(X^{\prime}\right)$ and $X^{A}\left(X^{\prime}\right)$ are known explicitly.

\section{ASYMPTOTICALLY ADS REISSNER-NORDSTRÖM SPACE-TIMES}

In this section we consider the thermodynamic geometry of charged, nonrotating AdS black holes. This was investigated in four-dimensional space-times by the authors of [9], and here the analysis is extended higher dimensional space-times with general dimension $D$.

With no extra work, and with a view to applications in the AdS/CFT correspondence, we can also incorporate more general event horizon topologies by denoting the curvature of the event horizon by $k$ : so $k=1$ is a spherical event horizon, $k=0$ a flat one (which we take to toroidal for convenience), and $k=-1$ the $(D-2)$-dimensional space of constant negative curvature.

The extensive variables are the electric charge $Q$ and the entropy $S$. The pressure $P$ will be kept fixed in this section, parametrized for notational convenience by

$$
\lambda=\frac{16 \pi P}{(D-1)(D-2)} .
$$

If $r_{h}$ is the radius of the outer horizon, the entropy, in units with $G=c=\hbar=1$, is

$$
S=\frac{\varpi r_{h}^{D-2}}{4}
$$

where $\varpi$ is the volume of the event horizon with $r_{h}$ set to unity. ${ }^{5}$ The mass (enthalpy) of the black hole is

$$
M=\frac{(D-2) \varpi r_{h}^{D-3}\left(k+\lambda r_{h}^{2}+\frac{Q^{2}}{r^{2 D-6}}\right)}{16 \pi}
$$

The Hawking temperature is

\footnotetext{
${ }^{5}$ For $k=+1$ this is the volume of the unit $(D-2)$ sphere, and for $k=0$ and $k=-1$ the event horizon can be made to have a finite area by suitably identifying points.
}

$$
T=\frac{(D-1) \lambda r_{h}^{2}+(D-3)\left(k-\frac{Q^{2}}{r_{h}^{2 D-6}}\right)}{4 \pi r_{h}},
$$

placing an upper bound $Q^{2} \leq\left(\frac{D-1}{D-3} \lambda r_{h}^{2}+k\right) r_{h}^{2 D-6}$ on $Q^{2}$ to ensure $T \geq 0$.

In [9] a Weinhold metric on the two-dimensional state space parametrized by $S$ and $Q$ using the Hessian of $M(S, Q, \lambda)$, with $\lambda$ fixed, was considered in $D=4$, with $X^{A}=(S, Q), A=1,2$,

$$
g_{A B}=\frac{\partial^{2} M}{\partial X^{A} \partial X^{B}}
$$

With $\lambda$ fixed this is a positive definite metric for a thermodynamically stable black hole. It gives rise to a geometry with a positive scalar curvature for $D=4$ [9], and this result generalizes to $D \geq 4$. The metric is written here in $\left(r_{h}, Q\right)$ coordinates, so as to avoid fractional powers of $S$ : using (14) it evaluates to

$$
g=\frac{(D-2) \varpi}{8 \pi}\left(\begin{array}{cc}
r_{h}^{D-5} Z_{Q}\left(r_{h}, Q, \lambda\right) & -\frac{(D-3) Q}{r_{h}^{D-2}} \\
-\frac{(D-3) Q}{r_{h}^{D-2}} & \frac{1}{r_{h}{ }^{D-3}}
\end{array}\right),
$$

where $Z_{Q}\left(r_{h}, Q, \lambda\right)$ is

$$
Z_{Q}\left(r_{h}, Q, \lambda\right)=(D-1) \lambda r_{h}^{2}-2 \pi r_{h} T+(D-3)^{2} \frac{Q^{2}}{r_{h}^{2 D-6}}
$$

The determinant of $g$ vanishes when the heat capacity diverges, but we need to be careful to distinguish between the heat capacity at constant charge $C_{Q}$ and the heat capacity at constant electric potential $C_{\Phi}$. In this context the electric potential is the thermodynamic conjugate of the charge,

$$
\Phi=\left.\frac{\partial M}{\partial Q}\right|_{S}=\frac{(D-2) \varpi Q}{8 \pi r_{h}^{D-3}}
$$

and the heat capacity at constant $\Phi$ is

$$
C_{\Phi}=\frac{(D-2) \varpi \pi r_{h}^{D-1} T}{2 Z_{\Phi}\left(r_{h}, Q, \lambda\right)},
$$

where

$$
Z_{\Phi}\left(r_{h}, Q, \lambda\right)=(D-1) \lambda r_{h}^{2}-2 \pi r_{h} T
$$

Indeed

$$
\operatorname{det} g=\frac{\varpi^{2}(D-2)^{2} Z_{\Phi}\left(r_{h}, Q, \lambda\right)}{64 \pi^{2} r_{h}^{2}}
$$


and a necessary condition for stability is

$$
(D-1) \lambda r_{h}^{2}>2 \pi r_{h} T,
$$

which is always true for $k \leq 0$ and only imposes a genuine constraint for $k=1$. The heat capacity at constant charge is

$$
C_{Q}=\frac{(D-2) \varpi \pi r_{h}^{D-1} T}{2 Z_{Q}\left(r_{h}, Q, \lambda\right)}
$$

and the analogue of $C_{P}>C_{V}$ for a gas is $C_{\Phi}>C_{Q}$. Since $Z_{Q}>Z_{\Phi}$, this is satisfied provided $Z_{\Phi}$ is not negative. Note that both $C_{Q}$ and $C_{\Phi}$ are finite for $k \leq 0$, and the heat capacity can become singular only if $k=1$.

The adiabatic electrical capacitance,

$$
\mathcal{C}_{S}=\left.\frac{\partial Q}{\partial \Phi}\right|_{S}=\frac{8 \pi r_{h}^{D-3}}{(D-2) \varpi}
$$

is independent of the electric charge, depending only on the entropy, and is always positive. The isothermal electrical capacitance $\mathcal{C}_{T}$ immediately follows from $C_{Q} \mathcal{C}_{T}=C_{\Phi} \mathcal{C}_{S}$.

There is a critical point if $\frac{\partial^{2} M}{\partial S^{2}}$ and $\frac{\partial^{2} M}{\partial S^{2}}$ vanish simultaneously. This cannot happen for $k=0$ or $k=-1$, but for $k=+1$ there is a critical point at

$$
r_{h, *}^{2}=\frac{(D-3)^{2}}{(D-1)(D-2) \lambda}, \quad \frac{Q_{*}^{2}}{r_{h, *}^{2 D-6}}=\frac{1}{(D-2)(2 D-5)},
$$

found in [41,42]. By definition $C_{Q}$ diverges at the critical point, but $C_{\Phi}$ is finite and negative there. Thus $C_{\Phi}<0$ at the critical point, which is therefore in a thermodynamically unstable regime unless $Q$ is held fixed.

The intrinsic scalar curvatures arising from the Weinhold and Ruppeiner geometries are described in detail in Appendix A. For the Weinhold metric (18) the Ricci scalar takes rather a simple form,

$$
R=\frac{(D-3)^{2}}{(D-2)} \frac{\pi r_{h} k}{S Z_{\Phi}^{2}}
$$

Thus the curvature of the event horizon is reflected in the sign of $R$; in particular the Weinhold metric is flat for $k=0$.

The curvature scalar associated with the corresponding Ruppeiner metric, with $\Lambda$ fixed, follows from the charged black hole analogue of (7) for a gas,

$$
\tilde{R}=T R-\left.\frac{1}{2} \frac{\partial}{\partial S}\right|_{Q} \ln \left(\frac{T C_{\Phi} \mathcal{C}_{S}}{Q^{2}}\right),
$$

or by direct calculation from the Ruppeiner metric. It is proportional to $\lambda$ and therefore vanishes in asymptotically flat space-time: explicitly
$\tilde{R}=\frac{(D-1) \lambda\left\{3 \pi T-(D-1) r_{h} \lambda\right\} \tilde{F}\left(r_{h}, \lambda, Q\right) C_{\Phi}^{2}}{2\{2 \pi(D-2) S T\}^{3}}$,

where

$$
\begin{aligned}
\tilde{F}\left(r_{h}, \lambda, Q\right)= & 2(D-1) \lambda r_{h}^{2}+4(D-4) \pi r_{h} T \\
& +2(D-3)^{2} \frac{Q^{2}}{r_{h}^{2 D-6}}
\end{aligned}
$$

is a positive function. That $\tilde{R}$ vanishes for asymptotically flat space-time in $D=4$ was observed in [9], and we see here that this statement generalizes to $D>4 . \tilde{R}$ can be of either sign when $\lambda>0$ and is negative for small temperatures, diverging to minus infinity for extremal black holes.

We see that a flat event horizon gives $R=0$ while a flat cosmology, $\Lambda=0$, gives $\tilde{R}=0$. It was observed in [25] that repulsive microscopic forces tend to give negative Ruppeiner curvature ${ }^{6}$ while attractive forces give positive curvature, but in the absence of an underlying theory of quantum gravity it is not at all clear whether this interpretation accounts for the changing sign of $\tilde{R}$ for ReissnerNordström black holes when $\lambda=\frac{3 \pi T}{(D-1) r_{h}}$.

The dependence of the Weinhold scalar on $k$ in (25) can also be given a thermodynamic interpretation. The sign of $k$ is significant for stability as there is no Hawking-Page phase transformation for $k \leq 0$. This can be seen by calculating the Gibbs free energy for the black hole,

$$
\mathcal{G}(T, \Phi)=M(S, Q)-T S-Q \Phi,
$$

which, in terms of $\lambda, r_{h}$, and $Q$, is

$$
\mathcal{G}=\frac{\varpi r_{h}^{D-3}}{16 \pi}\left(k-\lambda r_{h}^{2}-\frac{Q^{2}}{r_{h}^{2 D-3}}\right)
$$

The black hole is only stable against Hawking-Page decay if its Gibbs free energy is less than the Gibbs free energy of anti-de Sitter space filled with pure thermal radiation at the Hawking temperature of the black hole and, if the back reaction of the radiation is ignored, the latter is zero. Thus the black hole is stable against the Hawking-Page phase transition if the Gibbs free energy is negative, [2], which is always the case for $k \leq 0$. A Hawking-Page phase transition is possible only if $R>0$.

Note the critical point is not reflected in either of the curvature scalars because the singularities of $R$ and $\tilde{R}$ are determined by $\left(C_{Q} \mathcal{C}_{T}\right)^{2}=\left(C_{\Phi} \mathcal{C}_{S}\right)^{2}$ and there is a locus of zeros in the isothermal electrical capacitance $\mathcal{C}_{T}$ which

\footnotetext{
${ }^{6}$ Our convention for the sign of the Ricci scalar is that a sphere has positive curvature: this is opposite to that of [25].
} 
exactly cancels the singularities in $C_{Q}$. The fact that the analytic structure of the curvatures is determined by $C_{\Phi}$ and not by $C_{Q}$ is a phenomenon which also occurs for rotating black holes as we shall see in the next section. The same phenomenon is also present for the van der Waals gas where the singularities in the curvature are determined by $C_{P}$ and not $C_{V}$; the latter is in fact finite. For the van der Waals gas, however, the critical point is also determined by $C_{P}$ and not $C_{V}$, while for the black hole the critical point is determined by $C_{Q}$ and not $C_{\Phi}$, even though $C_{\Phi}$ can have singularities when $k=1$.

If the pressure is included as a thermodynamic variable, we should Legendre transform from $M(S, Q, P)$ to $U(S, Q, V)=M+P V$ to ensure a positive definite metric for thermodynamically stable black holes. However, the Weinhold and Ruppeiner metrics associated with the threedimensional state space, parametrized by $(S, Q, V)$, are singular because $S$ and $V$ are not independent for nonrotating black holes [43]. This restriction is removed when the black hole rotates, and we study this case in the next section.

\section{ASYMPTOTICALLY ADS MYERS-PERRY BLACK HOLES}

\section{A. Singly spinning black holes}

The thermodynamic state space associated with a $D$-dimensional rotating black hole is multidimensional in general, because of the increasing number of angular momenta as $D$ increases. For simplicity we set $Q=0$ and focus on the singly spinning case where only one of the $J^{i}$ is nonzero, $J^{1}=J \neq 0$ say, with $J^{i}=0$ for $i=2, \ldots, r$. The thermodynamic state space is then three-dimensional, parametrized by $(S, J, V)$. The geometry can be parametrized by the cosmological constant, a rotational parameter $a$, and the radius of the event horizon $r_{h}$.

Details are given in Appendix B, and here we summarize the relations between the geometric parameters and the thermodynamic ones. It is convenient to define dimensionless variables $\bar{a}=\frac{a}{r_{h}}$ and $\bar{\lambda}=\lambda r_{h}^{2}$ in terms of which the thermodynamic variables $(S, J, V)$ are [44,45]

$$
\begin{aligned}
S & =\frac{\varpi r_{h}^{D-2}}{4} \frac{\left(1+\bar{a}^{2}\right)}{\left(1-\bar{\lambda} a^{2}\right)} \\
J & =\frac{\varpi r_{h}^{D-2}}{8 \pi} \frac{\left(1+\bar{a}^{2}\right)(1+\bar{\lambda}) \bar{a}}{\left(1-\bar{\lambda} \bar{a}^{2}\right)^{2}}, \\
V & =\frac{\varpi r_{h}^{D-1}}{(D-1)(D-2)} \frac{\left(1+\bar{a}^{2}\right)\left\{D-2-(D-3) \bar{\lambda} \bar{a}^{2}+\bar{a}^{2}\right\}}{\left(1-\bar{\lambda} \bar{a}^{2}\right)^{2}},
\end{aligned}
$$

where $\varpi$ is the volume of a unit $(D-2)$-sphere. The conjugate variables $(T, \Omega, P)$ are

$$
\begin{aligned}
T & =\frac{(D-3)+(D-5) \bar{a}^{2}+\bar{\lambda}\left\{D-1+(D-3) \bar{a}^{2}\right\}}{4 \pi r_{h}\left(1+\bar{a}^{2}\right)}, \\
\Omega & =\frac{(1+\bar{\lambda}) \bar{a}}{\left(1+\bar{a}^{2}\right) r_{h}}, \\
P & =\frac{(D-1)(D-2) \bar{\lambda}}{16 \pi r_{h}^{2}},
\end{aligned}
$$

and the thermodynamic potentials are

$$
\begin{aligned}
M & =\frac{\varpi r_{h}^{D-3}}{16 \pi} \frac{\left(1+\bar{a}^{2}\right)(1+\bar{\lambda})\left\{D-2-(D-4) \bar{\lambda} \bar{a}^{2}\right\}}{\left(1-\bar{\lambda} \bar{a}^{2}\right)^{2}}, \\
U & =M-P V \\
& =\frac{\varpi r_{h}^{D-3}}{16 \pi} \frac{\left(1+\bar{a}^{2}\right)\left\{D-2-(D-3) \bar{\lambda} \bar{a}^{2}+\bar{\lambda}^{2} \bar{a}^{2}\right\}}{\left(1-\bar{\lambda} \bar{a}^{2}\right)^{2}} .
\end{aligned}
$$

All extensive quantities $(S, J, V, M$, and $U$ ) diverge when $\bar{\lambda} \bar{a}^{2}=1$, which should be viewed as the edge of thermodynamic state space [46]; in particular the entropy becomes negative for $\bar{\lambda} \bar{a}^{2}>1$ and only $\bar{\lambda} \bar{a}^{2}<1$ makes sense thermodynamically.

\section{B. Thermodynamic geometry}

\section{Fixed pressure}

For $\Lambda=0$ singly spinning black holes the thermodynamic state space is two-dimensional, the independent variables being either $S$ and $J$, for the Weinhold metric, or $M$ and $J$, for the Ruppeiner metric. The intrinsic curvatures associated with both the Weinhold and the Ruppeiner metrics were calculated in [15] and the Weinhold metric is flat for all $D$.

When $\Lambda<0$ and held fixed the space-time can be parametrized either by the geometric variables $\left(X^{A^{\prime}}\right)=$ $\left(r_{h}, \bar{a}\right)$ or by the thermodynamic variables $\left(X^{A}\right)=(S, J)$ : for calculations involving the thermodynamic metric the former are more useful because (28), (29), and (30) are simple ratios of polynomials of fairly low order in these variables which makes the algebra more tractable. With the explicit expressions (28) and (29) it is straightforward to evaluate the matrices $\frac{\partial X^{C}}{\partial X^{B^{\prime}}}$ and $\frac{\partial Y_{C}}{\partial X^{A^{A}}}$, with $Y_{C}=(T, \Omega)$, and hence determine the metric (14) and its curvature.

Details of the Weinhold and the Ruppeiner curvature scalars at fixed pressure are given in Appendix B. They diverge on the spinodal curve for the heat capacity at constant angular momentum, which is a

$$
C_{\Omega, P}=-\frac{4 \pi r_{h} T S\left\{D-2-(D-4) \bar{a}^{2}\right\}}{Z_{\Omega}(\lambda, \bar{a})}
$$

with 

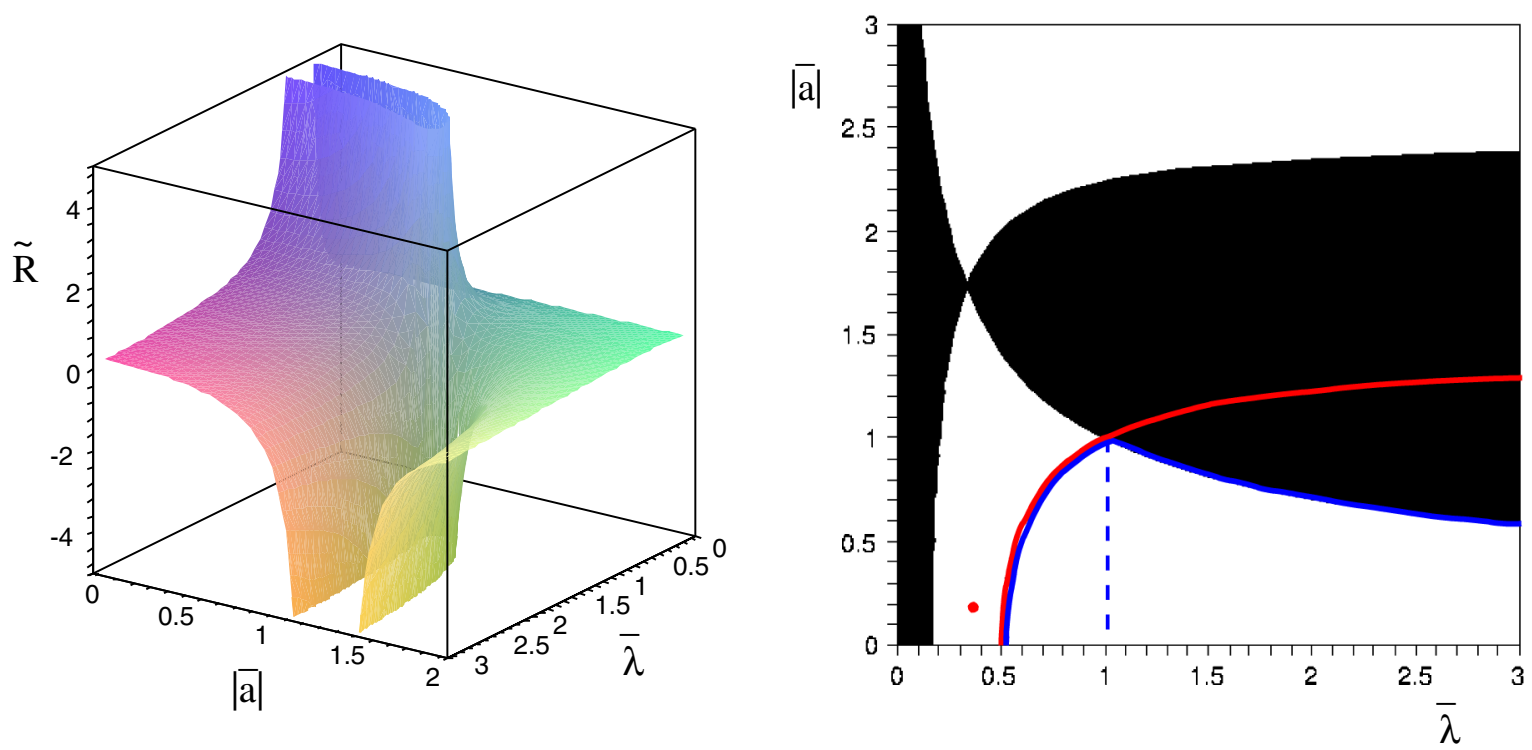

FIG. 1 (color online). The Ricci scalar for the Ruppeiner geometry associated with a singly spinning asymptotically AdS Myers-Perry black hole in $D=5$ at fixed $\bar{\lambda}$, as a function of $\bar{\lambda}$ and $\bar{a}$ (multiplied by $r_{h}^{3}$ to render it dimensionless). In the right-hand figure $\tilde{R}$ is positive in the white regions and negative in the black regions. The regime of local thermodynamical stability [determined by convexity $M(S, J, P)$ with $P$ fixed] lies below the blue curve, bounded by the hyperbola $\bar{\lambda} \bar{a}^{2}=1$ (blue) and the spinodal curve $Z_{\Omega}=0$ (red). The vertical dashed line indicates the Hawking-Page phase transition-the black hole is stable against the Hawking-Page phase transition only for $\bar{\lambda}>1$. The critical point lies in the unstable regime if the angular momentum is not fixed and is indicated by the dot.

$$
Z_{\Omega}(\bar{\lambda}, \bar{a})=(D-3)\left(1+\bar{\lambda} \bar{a}^{2}\right)-(D-1) \bar{\lambda}-(D-5) \bar{a}^{2}
$$

The spinodal curve, $Z_{\Omega}=0$, is therefore given by

$$
\bar{\lambda}(\bar{a})=\frac{(D-3)-(D-5) \bar{a}^{2}}{(D-1)-(D-3) \bar{a}^{2}}:=\bar{\lambda}_{\infty}
$$

In contrast the heat capacity at constant angular momentum is

$$
C_{J}=-\frac{4 \pi r_{h} T S\left(1+\bar{a}^{2}\right)^{2}\left\{D-2+(D-4) \lambda \bar{a}^{2}\right\}}{Z_{J}(\bar{\lambda}, \bar{a})},
$$

where $Z_{J}$ is quadratic in $\lambda$ [the explicit expression is given explicitly in Appendix B, Eq. (B7)]. In the thermodynamically stable regime $C_{\Omega}>C_{J}$.

There is a critical point and a second order phase transition associated with fixed $J$, first found in [47] for $D=4$ but which has an analogue in any $D$. This critical point is not visible for fixed $\Omega$ as $C_{\Omega}$ is finite there and, in parallel with the Reissner-Nordström case, $C_{\Omega}$ is in fact negative at the critical point.

Explicitly the Weinhold curvature is

$$
R=\frac{16 \pi \bar{\lambda}\left(1-\bar{\lambda} \bar{a}^{2}\right) F_{1}(\bar{\lambda}, \bar{a})}{\varpi r_{h}^{D-3}\left(1+\bar{a}^{2}\right)\left\{D-2+(D-4) \bar{\lambda} \bar{a}^{2}\right\}^{2} Z_{\Omega}^{2}(\bar{\lambda}, \bar{a})}
$$

with $F_{1}(\bar{\lambda}, \bar{a})$ a polynomial linear in $\bar{\lambda}$ and quartic in $\bar{a}^{2}$, given explicitly in Appendix B, Eq. (B4), while the Ruppeiner curvature is

$$
\tilde{R}=-\frac{(D-3)\left(1-\bar{\lambda}^{2} \bar{a}^{4}\right) \tilde{F}_{1}(\bar{\lambda}, \bar{a}) \tilde{F}_{2}(\bar{\lambda}, \bar{a})}{\pi \varpi r_{h}^{D-1}\left(1+\bar{a}^{2}\right)^{2} T\left\{D-2+(D-4) \bar{a}^{2}\right\} Z_{\Omega}^{2}(\bar{\lambda}, \bar{a})},
$$

with $\tilde{F}_{1}(\bar{\lambda}, \bar{a})$ a linear in $\bar{\lambda}$ and $\bar{a}^{2}$, and $\tilde{F}_{2}(\bar{\lambda}, \bar{a})$ quadratic in $\bar{\lambda}$ and $\bar{a}^{2}$ [Appendix B, Eqs. (B10) and (B11)].

The Weinhold metric is no longer flat when $\Lambda<0$, for any $D \geq 4$, and can be of either sign. Both the Weinhold and the Ruppeiner metrics are flat on the line $\bar{\lambda} \bar{a}^{2}=1$, which is the boundary of the region where a thermodynamic interpretation of the black hole is consistent as the entropy, angular momentum, and mass all diverge on this line. Both are singular on the spinodal curve for the heat capacity at constant angular velocity (31).

As an example the Ricci scalar for the Ruppeiner metric is shown in Fig. 1, for $D=5$ (scaled by $r_{h}^{3}$ to make it dimensionless). It is negative for small $\bar{\lambda}$, for any value of the rotation parameter $\bar{a}$, but is positive everywhere in the thermodynamically stable regime. The latter observation is also true in $D=4[18,19,21]$.

The singularities in both the Weinhold and the Ruppeiner curvatures are determined by those of $C_{\Omega}$ and not $C_{J}$. In the context of rotating black holes we have $C_{J} \mathcal{I}_{T}=C_{\Omega} \mathcal{I}_{S}$, where $\mathcal{I}_{S}$ and $\mathcal{I}_{T}$ are the isentropic and isothermal moments of inertia, respectively, Eqs. (B7) and (B8) in 

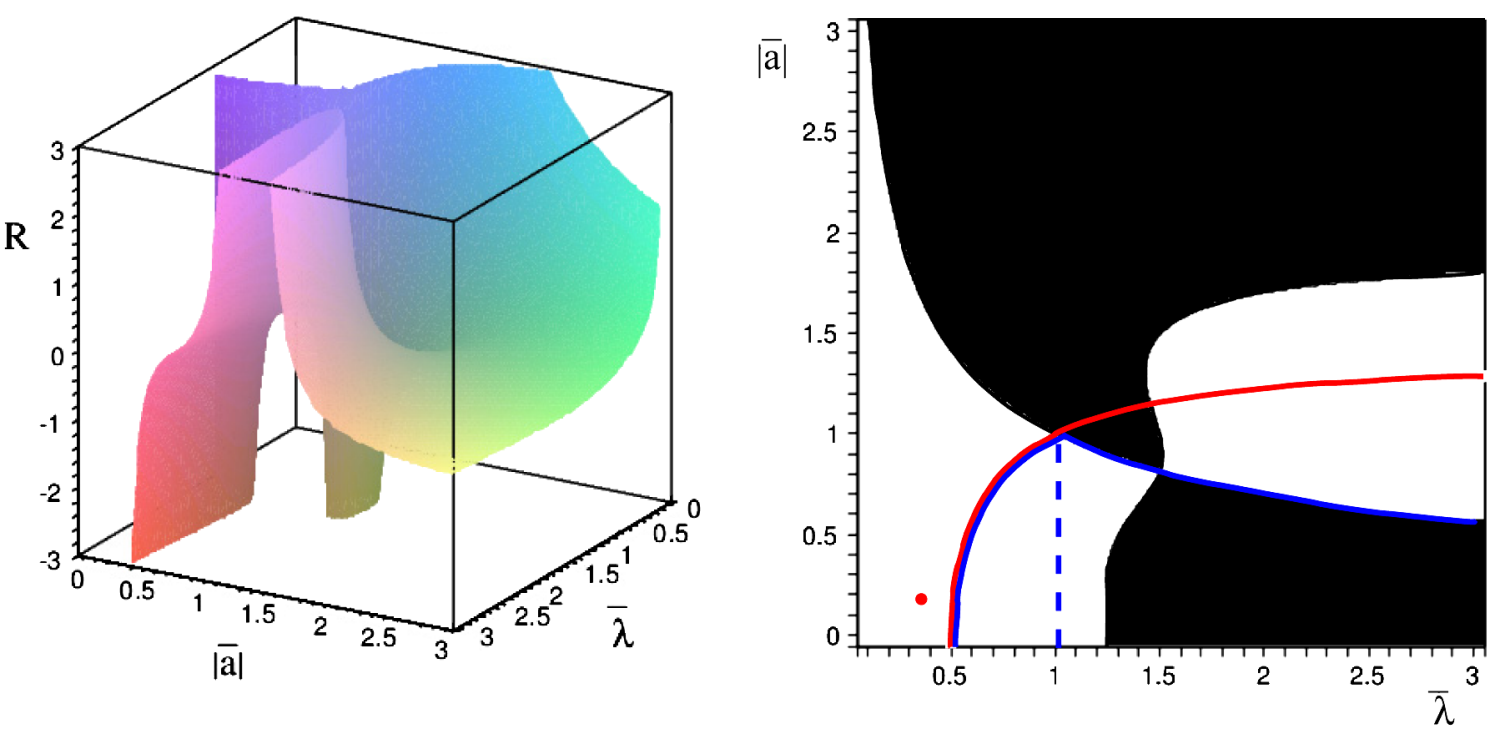

FIG. 2 (color online). The Ricci scalar for the Weinhold geometry associated with a singly spinning asymptotically AdS Myers-Perry black hole in $D=5$, as a function of $\bar{\lambda}$ and $\bar{a}$ (multiplied by $r_{h}^{2}$ to render it dimensionless). The color coding is the same as in 1 .

Appendix B. The curvatures here are proportional to $\left(C_{J} \mathcal{I}_{T}\right)^{2}=\left(C_{\Omega} \mathcal{I}_{S}\right)^{2}$ : there are singularities in $C_{J}$ that are canceled by corresponding zeros in the isothermal moment of inertia ${ }^{7} \mathcal{I}_{T}$ and singularities in $\mathcal{I}_{T}$ which are the same as those in $C_{\Omega}$. Note also that the singularities of

$\mathcal{I}_{S}=\left.\frac{\partial J}{\partial \Omega}\right|_{S}=\frac{r_{h} S\left(1+a^{2}\right)^{2}\left\{D-2+(D-4) \lambda \bar{a}^{2}\right\}}{2 \pi\left(1-\bar{\lambda} \bar{a}^{2}\right)^{2}\left\{D-2-(D-4) \bar{a}^{2}\right\}}$,

in the region with $\bar{\lambda} \bar{a}^{2}<1$, are canceled by zeros in $C_{\Omega}$, and the only singularities in the curvatures are those of $C_{\Omega}$. For $D \geq 5, C_{\Omega}$ is positive if either $Z_{\Omega}>0$ and $a^{2}<\frac{D-2}{D-4}$ or $Z_{\Omega}<0$ and $\bar{a}^{2}>\frac{D-2}{D-4}$, but the latter possibility necessarily implies that $\mathcal{I}_{S}<0$ : for ultraspinning black holes the moment of inertia is negative.

In summary, when the pressure is held fixed, both the Weinhold and the Ruppeiner scalar curvatures diverge on the spinodal curve for $C_{\Omega}, Z_{\Omega}=0$ in (32), and these are the only singularities. The curvature singularities are determined by those of $C_{\Omega}$, not $C_{J}$.

\section{Varying pressure}

Allowing for varying $\Lambda$ there is potentially a fourdimensional space of thermodynamic states associated with singly spinning charged black holes, parametrized by $(S, Q, J, V)$. A completely general analysis would be rather complicated, and we restrict ourselves here to studying the three-dimensional state space of singly spinning electrically neutral black holes. Even then, with a

\footnotetext{
${ }^{7}$ In this section $\lambda$ is held fixed, so it is implicit that the moment of inertia here is calculated at fixed pressure $\mathcal{I}_{T}=\mathcal{I}_{T, P}$.
}

three-dimensional state space, the full Ricci tensor is necessary for a complete description of the curvature, and for simplicity the discussion here is restricted to the Ricci scalar, for which the properties are not too difficult to extract.

The diagonal components of the Weinhold metric in thermodynamic coordinates $(S, J, V)$ have direct physical interpretations,

$$
\begin{aligned}
g_{S S} & =\frac{\partial T}{\partial S}=\frac{T}{C_{V, J}}, \\
g_{J J} & =\frac{\partial \Omega}{\partial J}=\mathcal{I}_{S, V}^{-1}, \\
g_{V V} & =-\frac{\partial P}{\partial V}=\frac{1}{V \kappa_{S, J}},
\end{aligned}
$$

where $\mathcal{I}_{S, V}$ is the isentropic moment of inertia of the black hole, with $V$ fixed.

The Weinhold and Ruppeiner metrics of a singly spinning asymptotically AdS Myers-Perry black hole, with internal energy $U(S, J, V)$, can be evaluated in $\left(r_{h}, \bar{a}, \bar{\lambda}\right)$ coordinates using (14). They are found to be

$$
R=\frac{16 \pi}{(D-3) \varpi r_{h}^{D-3}} \frac{\left(1-\bar{\lambda} \bar{a}^{2}\right) F_{2}(\bar{\lambda}, \bar{a})}{\bar{a}^{4}\left(1+\bar{a}^{2}\right)(1+\bar{\lambda})^{2} Z_{\Omega}^{2}(\bar{\lambda}, \bar{a})},
$$

where $F_{2}(\bar{\lambda}, \bar{a})$ is quadratic in $\bar{\lambda}$ and quartic in $\bar{a}^{2}$ (more details are given in Appendix B).

$$
\tilde{R}=\frac{\left(1-\bar{\lambda} \bar{a}^{2}\right) \tilde{F}_{3}(\bar{\lambda}, \bar{a})}{4 \pi(D-3) \varpi r_{h}^{D-1} \bar{a}^{4}\left(1+\bar{a}^{2}\right)^{3}(1+\bar{\lambda})^{2} T Z_{\Omega}^{2}(\bar{\lambda}, \bar{a})},
$$



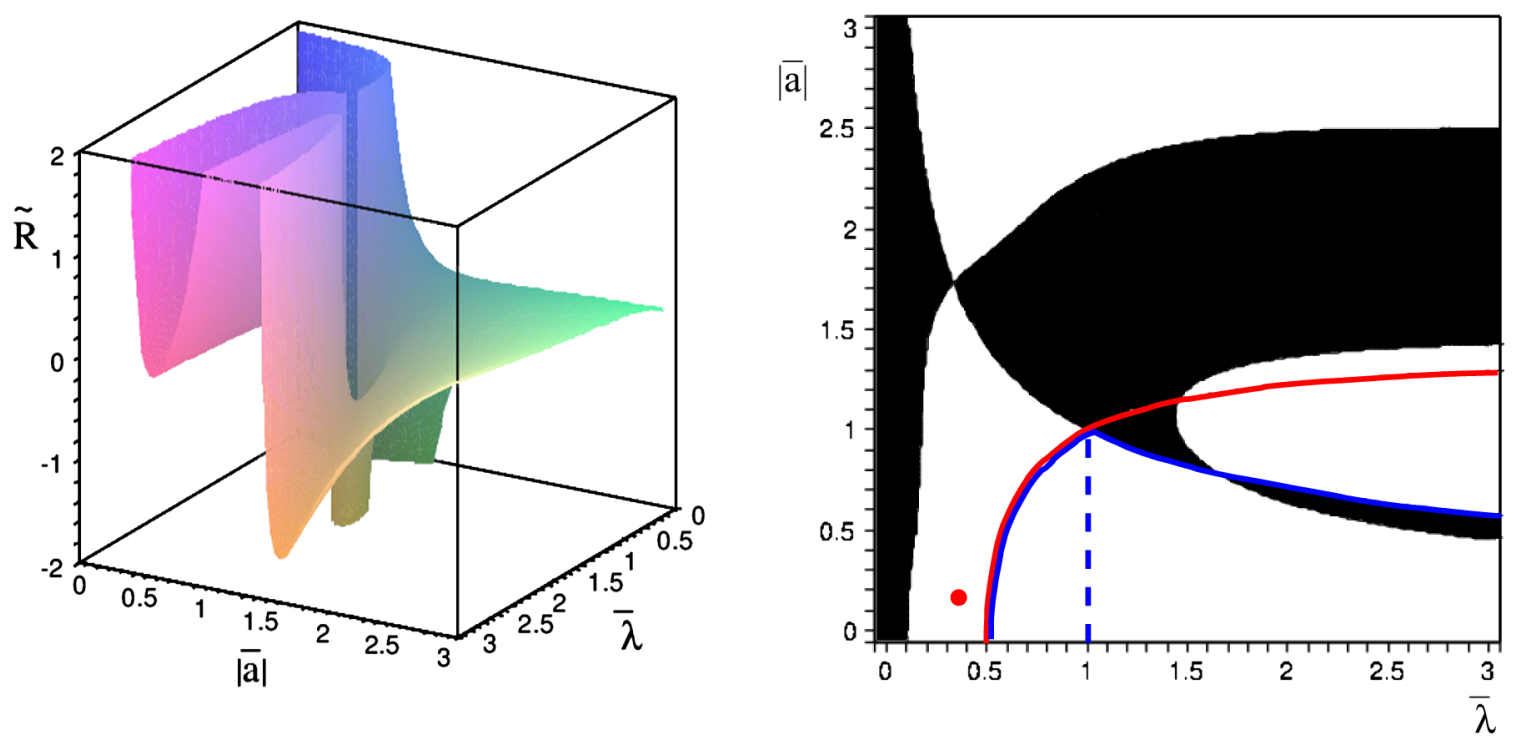

FIG. 3 (color online). The Ricci scalar for the Ruppeiner geometry associated with a singly spinning asymptotically AdS Myers-Perry black hole in $D=5$, as a function of $\bar{\lambda}$ and $\bar{a}$ (multiplied by $r_{h}^{3}$ to render it dimensionless). The color coding is the same as in Fig. 1 .

where $\tilde{F}_{3}\left(\bar{\lambda}, \bar{a}^{2}\right)$ is a quintic polynomial in $\bar{\lambda}$ and sixth order in $\bar{a}^{2}$.

Both curvatures diverge for $\bar{a}^{2}=0$ and on the curve $\bar{\lambda}_{\infty}$. The former singularity, at $\bar{a}^{2}=0$, is associated with the incompressibility of a nonrotating black hole [48] while the latter is again the same locus of points as that on which the heat capacity at constant angular velocity and pressure $C_{\Omega, P}$ diverges [38].

For illustrative purposes we show the Weinhold Ricci scalar, $R$, for $D=5$ in Fig. 2, scaled by a factor $r_{h}^{2}$ to make it a dimensionless function of only two variables, $\bar{a}$ and $\bar{\lambda}$. For comparison the Ruppeiner Ricci scalar $\tilde{R}$ is shown in Fig. 3, scaled by $r_{h}^{3}$. In both cases the Ricci scalar diverges on the curve $\bar{\lambda}_{\infty}$ in Eq. (33), where the denominator has a double zero associated with a singularity in the heat capacity. The Ricci scalar also diverges for $\bar{a}=0$, where the black hole is incompressible. A correlation between singularities in response functions and singularities in the Ricci scalar for thermodynamic state space is a generic feature of the geometry of black hole thermodynamic state space [6], and for black holes in particular [26]. Both Ricci scalars vanish on the hyperbola $\bar{\lambda} \bar{a}^{2}=1$, except at the point $\bar{\lambda}=\bar{a}^{2}=1$, since the denominators of both $R$ and $\tilde{R}$ have double zeros there [Eqs. (B16) and (B18) in Appendix B].

The region of thermodynamic stability lies below the solid blue curve in the right-hand plots in Figs. 2 and 3 (the black hole is also unstable against the Hawking-Page phase transition for $\bar{\lambda}<1$ ). The thermodynamically stable regime is isolated from the divergences in the heat capacity, except at the single point $\bar{a}^{2}=\bar{\lambda}=1$. The Ruppeiner scalar is positive close to the line of the Hawking-Page phase transition, but can become negative for large pressure and entropy.
Unlike the two parameter case the Ricci scalar does not carry complete information about the curvature for a threedimensional state space, such as the one considered in this section. The Ricci tensor $R_{A B}$ does, however, have complete information, and a second invariant is given by the trace of its square, $R_{A B} R^{A B}$, and this can also be evaluated. ${ }^{8}$ For both the Weinhold metric and the Ruppeiner this quadratic invariant has the same singularity structure as the square of the Ricci scalar squared: for the Weinhold metric

$$
R_{A B} R^{A B}=\frac{64 \pi^{2}\left(1-\bar{\lambda} \bar{a}^{2}\right)^{2} P_{5}(\bar{\lambda}, \bar{a})}{(D-3)^{2} \varpi^{2} r_{h}^{2(D-3)} \bar{a}^{8}\left(1+\bar{a}^{2}\right)^{2}(1+\bar{\lambda})^{4} Z_{\Omega}^{4}(\bar{\lambda}, \bar{a})},
$$

with $P_{5}$ a polynomial quintic in $\bar{\lambda}$ and of order 8 in $\bar{a}^{2}$, with integer coefficients, while for the Ruppeiner metric

$$
\begin{aligned}
& \tilde{R}_{A B} \tilde{R}^{A B} \\
& =\frac{\left(1-\bar{\lambda} \bar{a}^{2}\right)^{2} P_{10}(\bar{\lambda}, \bar{a})}{16 \pi^{2}(D-3)^{2} \varpi^{2} r_{h}^{2(D-1)} \bar{a}^{8}\left(1+\bar{a}^{2}\right)^{6}(1+\bar{\lambda})^{4} T^{2} Z_{\Omega}^{4}(\bar{\lambda}, \bar{a})}
\end{aligned}
$$

with $P_{10}$ of order 10 in $\bar{\lambda}$ and order 12 in $\bar{a}^{2}$, with integer coefficients.

\section{CONCLUSIONS}

The thermodynamic geometry of asymptotically anti-de Sitter black holes in $D$ space-time dimensions has been

\footnotetext{
${ }^{8}$ I am grateful to the referee for the suggestion of investigating this quadratic invariant.
} 
examined in three special cases: electrically charged static black holes with fixed cosmological constant; electrically neutral singly spinning asymptotically AdS Myers-Perry black holes with fixed cosmological constant; and electrically neutral singly spinning asymptotically AdS MyersPerry black holes in an extended state space in which the cosmological constant is included among the thermodynamic variables. The first two cases generalize previous studies in the $D=4$ case to arbitrary dimension, while the last is a new direction in the geometry of black hole thermodynamics in which the black hole volume is interpreted as the thermodynamic variable conjugate to the positive pressure supplied by a negative cosmological constant. The analysis is based on Eq. (14) which expresses the thermodynamic metric in a manifestly general coordinate covariant way, allowing any convenient coordinate system to be used for the calculations.

Our first observation extends the analysis of [9] for four-dimensional spherical black holes to $D$ space-time dimensions and also to event horizons with positive $(k=+1)$, negative $(k=-1)$, and vanishing $(k=0)$ curvature. At fixed $\Lambda \leq 0$ the sign of the Weinhold curvature, derived from $M(S, Q, P)$ for a charged, nonrotating black hole in $D$ space-time dimensions, is determined by the topology of the event horizon: the Weinhold curvature is positive for spherical event horizons and negative for hyperbolic horizons, while flat event horizons give a flat Weinhold metric. This behavior shows a correlation with thermodynamic stability, as only $k=1$ black holes can support a Hawking-Page phase transition. In contrast, the Ruppeiner metric is flat for $\Lambda=0$ but can be of either sign for $\Lambda<0$

For spherical event horizons the black hole can rotate and the Weinhold metric on the two-dimensional state space of singly spinning electrically neutral black holes, derived from $M(S, J, P)$ with $P$ fixed, is flat when $\Lambda=0$.

For asymptotically AdS black holes which are both charged and spinning there is a critical point for fixed $Q$ and $J$ [47], but not for fixed $\Phi$ or fixed $\Omega$. General considerations suggest that the thermodynamic curvatures should diverge on spinodal curves, but the analysis in Sec. III and Sec. IV shows that the thermodynamic curvatures do not diverge on the singularities of $C_{Q}$ or $C_{J}$. Rather, because of cancellations in $C_{Q} \mathcal{C}_{T}=C_{\Phi} \mathcal{C}_{S}$ and $C_{J} \mathcal{I}_{T}=C_{\Omega} \mathcal{I}_{S}$, they diverge on the singularities of $C_{\Phi}$ and $C_{\Omega}$. Contrary to expectations the thermodynamic curvatures do not diverge at the critical points. This unusual feature of the geometry of the thermodynamic state space for black holes may be due to the inhomogeneous scaling of the thermodynamic variables that makes the Smarr different from the integrated form of the Gibbs-Duhem relation.

For singly spinning electrically neutral black holes the thermodynamic state space can be enhanced to include the volume as a thermodynamic variable. The internal energy is a function of volume rather than pressure, $U(S, J, V)$ where a negative cosmological constant provides a positive pressure and $V=\left.\frac{\partial M}{\partial P}\right|_{S, J}$, with $M$ the black hole mass, is the thermodynamic volume. Unlike the mass $M(S, J, P)$, which is the enthalpy in thermodynamics, the internal energy $U(S, J, V)$ is a concave function of all its arguments for thermodynamically stable systems and gives rise to a positive definite Weinhold and Ruppeiner metrics in the region of parameter space where the black hole is thermodynamically stable. The resulting Ricci scalars have been calculated and shown to diverge for $J=0$, when the black hole is incompressible, and when the heat capacity at constant angular velocity and pressure, $C_{\Omega . P}$, diverges. Again the critical point is not visible in either the Weinhold or the Ruppeiner curvature scalars.

Symbolic manipulations performed in this analysis were carried out using Maple ${ }^{\mathcal{C}}$.

\section{APPENDIX A: THERMODYNAMIC CURVATURE OF CHARGE BLACK HOLES}

For nonrotating, asymptotically AdS black holes, the space-time metric is

$$
d s^{2}=-f(r) d t^{2}+\frac{1}{f(r)} d r^{2}+r^{2} d^{2} \Omega_{(D-2)},
$$

where $d^{2} \Omega_{(D-2)}$ is the line element on a unit $(D-2)$ dimensional sphere, torus, or hyperbolic space for $k=1,0$, or -1 , respectively, and

$$
f(r)=k-\frac{2 \mu}{r^{D-3}}+\frac{Q^{2}}{r^{2 D-6}}+\lambda r^{2} .
$$

The mass and the entropy are

$$
M=\frac{(D-2) \varpi \mu}{8 \pi}=\frac{(D-2) \varpi r_{h}^{D-3}\left(k+\frac{Q^{2}}{r_{h}^{2 D-6}}+r_{h}^{2} \lambda\right)}{16 \pi}
$$

and

$$
S=\frac{\varpi r_{h}^{D-2}}{4} .
$$

$Q$ is the electric charge and $\lambda=-\frac{2 \Lambda}{(D-1)(D-2)}$.

The entropy and the Hawking temperature are

$$
S=\frac{\varpi}{4} r_{h}^{D-2}
$$

and

$$
T=\frac{f^{\prime}\left(r_{h}\right)}{4 \pi}=\frac{(D-1) \lambda r_{h}^{2}+(D-3)\left(k-\frac{Q^{2}}{r_{h}^{2 D-6}}\right)}{4 \pi r_{h}},
$$


where $\varpi$ is the volume of a unit $(D-2)$ sphere, torus, or hyperbolic space for $k=1,0$, or $-1 .^{9}$ For fixed $\Lambda<0$, the Weinhold metric is the $2 \times 2$ Hessian matrix obtained by differentiating $M(S, Q, P)$ with respect to $S$ and $Q$, keeping $P$ fixed,

$$
g=\frac{\varpi(D-2)}{8 \pi}\left(\begin{array}{cc}
r_{h}^{D-5} Z_{Q}\left(r_{h}, \lambda, Q\right) & -\frac{(D-3) Q}{r_{h}^{D-2}} \\
-\frac{(D-3) Q}{r_{h}^{D-2}} & \frac{1}{r_{h}^{D-3}}
\end{array}\right),
$$

where

$$
\begin{aligned}
Z_{Q}\left(r_{h}, \lambda, Q\right)= & \frac{1}{2}\left\{(D-1) r_{h}{ }^{2} \lambda-(D-3) k\right. \\
& \left.+(D-3)(2 D-5) \frac{Q^{2}}{r_{h}{ }^{2 D-6}}\right\} .
\end{aligned}
$$

$Z_{Q}\left(r_{h}, \lambda, Q\right)$ is of course related to the heat capacity at constant charge and pressure,

$$
C_{Q, P}=\frac{(D-2) \pi \varpi r_{h}^{D-1} T}{2 Z_{Q}\left(r_{h}, Q, \lambda\right)} .
$$

The zero locus of $Z_{Q}\left(r_{h}, \lambda, Q\right)$ is the spinodal curve of $C_{Q, P}$. This is, however, not visible in the determinant,

$$
\operatorname{det} g=\frac{\varpi^{2}(D-2)^{2} Z_{\Phi}\left(r_{h}, Q, \lambda\right)}{64 \pi^{2} r_{h}^{2}},
$$

where

$$
Z_{\Phi}\left(r_{h}, Q, \lambda\right)=\frac{1}{2}\left\{(D-1) r_{h}^{2} \lambda-(D-3)\left(k-\frac{Q^{2}}{r_{h}^{2 D-6}}\right)\right\} .
$$

The determinant reflects the singularity structure of the heat capacity at constant electric potential, $\Phi=\left.\frac{\partial M}{\partial Q}\right|_{S, P}=$ $\frac{(D-2) \varpi Q}{8 \pi r_{h}^{D-3}}$, which is

$$
C_{\Phi, P}=\frac{(D-2) \pi \varpi r_{h}^{D-1} T}{2 Z_{\Phi}\left(r_{h}, Q, \lambda\right)} .
$$

The Weinhold scalar curvature is

$$
R=\frac{4 \pi k(D-3)^{2}}{(D-2) \varpi r_{h}^{D-3} Z_{\Phi}\left(r_{h}, Q, \lambda\right)^{2}} .
$$

For $k=1$ the singularities in the curvature match those of the heat capacity of the black hole at constant pressure, $C_{\Phi, P}$. For $k<0, C_{\Phi, P}$ and $R$ are finite and $R$ is negative.

\footnotetext{
${ }^{9}$ For $k=0$ and -1 the event horizon volume can be made finite by identifying points.
}

The Weinhold curvature scalar can be succinctly written in terms of the electrical capacitance,

$$
\mathcal{C}_{S, P}=\left.\frac{\partial Q}{\partial \Phi}\right|_{S, P}=\frac{8 \pi r_{h}^{D-3}}{(D-2) \varpi},
$$

and the entropy (A5) as

$$
R=\frac{(D-3)^{2}}{(D-2)} \frac{\pi r_{h} k}{S Z_{\Phi}^{2}}
$$

The Ruppeiner metric, on the other hand, based on the Hessian matrix of $S(M, Q, P)$ with a two-dimensional state space consisting of $(M, Q)$ and $P$ fixed, has scalar curvature

$$
\tilde{R}=\frac{4(D-1) \lambda\left(3 \pi T-(D-1) r_{h} \lambda\right) \tilde{F}\left(\lambda, r_{h}, Q\right) C_{\Phi, P}^{2}}{\left\{(D-2) \varpi r_{h}^{D-2} \pi T\right\}^{3}},
$$

where $\tilde{F}\left(\lambda, r_{h}, Q\right)$ is a linear function of $\lambda$,

$$
\begin{aligned}
\tilde{F}\left(\lambda, r_{h}, Q\right)= & (D-1)(D-2) r_{h}^{2} \lambda \\
& +(D-3)\left\{(D-4) k+(D-2) \frac{Q^{2}}{r_{h}^{2 D-6}}\right\},
\end{aligned}
$$

which is positive for $k=1$.

\section{APPENDIX B: METRIC AND RICCI SCALAR FOR ROTATING BLACK HOLES}

A singly spinning asymptotically AdS Myers-Perry black hole, in $D>3$ dimensions, has the line element [46]

$$
\begin{aligned}
d s^{2}= & -\frac{\Delta}{\rho^{2}}\left(d t-\frac{a}{\Xi} \sin ^{2} \theta d \phi\right)^{2}+\frac{\rho^{2}}{\Delta} d r^{2}+\frac{\rho^{2}}{\Delta_{\theta}} d \theta^{2} \\
& +\frac{\Delta_{\theta} \sin ^{2} \theta}{\rho^{2}}\left(a d t-\frac{r^{2}+a^{2}}{\Xi} d \phi\right)^{2}+r^{2} \cos ^{2} \theta d \Omega_{(D-2)},
\end{aligned}
$$

where

$$
\begin{aligned}
\Delta & =\left(r^{2}+a^{2}\right)\left(1+\lambda r^{2}\right)-\frac{2 \mu}{r^{D-5}}, \\
\Delta_{\theta} & =1-\lambda a^{2} \cos ^{2} \theta, \\
\rho^{2} & =r^{2}+a^{2} \cos ^{2} \theta, \\
\Xi & =1-\lambda a^{2} .
\end{aligned}
$$

$\mu, a$, and $\lambda$ are geometric parameters related to mass, rotation, and cosmological constant, respectively (as before, $\left.\lambda=-\frac{2 \Lambda}{(D-1)(D-2)}\right)$. There is an event horizon at the largest root, $r_{h}$, of 


$$
\Delta\left(r_{h}\right)=0 \Rightarrow \mu=\frac{1}{2} r_{h}^{D-5}\left(r_{h}^{2}+a^{2}\right)\left(1+\lambda r_{h}^{2}\right) .
$$

The Hawking temperature is

$$
T=\frac{(D-3)\left(1+\lambda a^{2}\right) r_{h}^{2}+(D-1) \lambda r_{h}^{4}+(D-5) a^{2}}{4 \pi r_{h}\left(r_{h}^{2}+a^{2}\right)} ;
$$

for $D>4$ this is a positive function for any $a$. The condition that $a^{2} \geq 0$ forces $T$ to lie in the range

$$
(D-3) \lambda r_{h}^{2}+(D-5) \leq 4 \pi r_{h} T \leq(D-1) \lambda r_{h}^{2}+(D-3) \text {. }
$$

When $\Lambda$ is fixed, the Weinhold metric derived from $M(S, J, P)$ with fixed $P$ is, in $\left(r_{h}, a\right)$ coordinates,

$$
g=\frac{\varpi r_{h}^{D-5}}{16 \pi\left(r_{h}^{2}+a^{2}\right)}\left(\begin{array}{cc}
\frac{p_{1}\left(r_{h}, \lambda, a\right)}{\left(1-\lambda a^{2}\right)} & -\frac{2 a\left(1-\lambda^{2} r_{h}^{4}\right)\left((D-1) r_{h}^{2}+(D-5) a^{2}\right)}{r_{h}\left(1-\lambda a^{2}\right)^{2}} \\
-\frac{2 a\left(1-\lambda^{2} r_{h}^{4}\right)\left((D-1) r_{h}^{2}+(D-5) a^{2}\right)}{r_{h}\left(1-\lambda a^{2}\right)^{2}} & \frac{2\left(1+\lambda r_{h}^{2}\right)^{2}\left[\left(3-a^{2}\right) \lambda a^{2}+r_{h}^{2}-3 a^{2}\right]}{\left(1-\lambda a^{2}\right)^{3}}
\end{array}\right),
$$

where $p_{1}\left(r_{h}, \lambda, a\right)$ in the top-left entry is

$$
\begin{aligned}
p_{1}\left(r_{h} \lambda, a\right)= & \lambda\left[(D-3)(D-4) a^{4}+2\left(D^{2}-5 D+3\right) a^{2} r_{h}^{2}\right. \\
& \left.+(D-1)(D-2) r_{h}^{4}\right]-(D-4)(D-5) a^{4} \\
& -2\left(D^{2}-7 D+9\right) a^{2} r_{h}^{2}-(D-2)(D-3) r_{h}^{4} .
\end{aligned}
$$

The Ricci scalar arising from this Weinhold metric is, in terms of dimensionless variables $\bar{\lambda}=\lambda r_{h}^{2}$ and $\bar{a}=\frac{a}{r_{h}}$,

$$
R=\frac{16 \pi \bar{\lambda}\left(1-\bar{\lambda} \bar{a}^{2}\right) F_{1}(\bar{\lambda}, \bar{a})}{\varpi r_{h}^{D-3}\left(1+\bar{a}^{2}\right)\left\{D-2+(D-4) \bar{\lambda} \bar{a}^{2}\right\}^{2} Z_{\Omega}^{2}(\bar{\lambda}, \bar{a})},
$$

where

$$
Z_{\Omega}(\bar{\lambda}, \bar{a})=(D-3)\left(1+\bar{\lambda} \bar{a}^{2}\right)-(D-1) \bar{\lambda}-(D-5) \bar{a}^{2},
$$

and $F_{1}(\bar{\lambda}, \bar{a})$ is linear in $\bar{\lambda}$,

$$
F_{1}(\bar{\lambda}, \bar{a})=A_{1}(\bar{a}) \bar{a}^{2} \bar{\lambda}+B_{1}(\bar{a}),
$$

with

$$
\begin{aligned}
A_{1}(\bar{a})= & (D-5)^{2} \bar{a}^{4}\left\{(D-4) \bar{a}^{2}+(D-6)\right\} \\
& -(D-1)\left(D^{2}-17 D+48\right) \bar{a}^{2}-(D-1)^{2}(D-2), \\
B_{1}(\bar{a})= & -(D-5) \bar{a}^{4}\left\{(D-4)(D-5) \bar{a}^{2}\right. \\
& \left.+\left(D^{2}+5 D-18\right)\right\}+(D-1)^{2}\left\{D \bar{a}^{2}+(D-2)\right\} .
\end{aligned}
$$

The zeros of $Z_{\Omega}$ reflect singularities in the heat capacity, in this instance the heat capacity at constant angular velocity and pressure,

$$
C_{\Omega, P}=-\frac{4 \pi r_{h} T S\left\{D-2-(D-4) \bar{a}^{2}\right\}}{Z_{\Omega}(\bar{\lambda}, \bar{a})} .
$$

The heat capacity at constant angular momentum and pressure has a rather different structure,

$$
C_{J, P}=-\frac{4 \pi r_{h} T S\left(1+\bar{a}^{2}\right)^{2}\left\{D-2+(D-4) \lambda \bar{a}^{2}\right\}}{Z_{J}(\bar{\lambda}, \bar{a})}
$$

with

$$
\begin{aligned}
Z_{J}(\bar{\lambda}, \bar{a})= & -\bar{\lambda}^{2} \bar{a}^{2}\left\{(D-3) \bar{a}^{4}+6 \bar{a}^{2}+3(D-1)\right\} \\
& +\bar{\lambda}\left\{(D-5) \bar{a}^{6}+(5 D-33) \bar{a}^{4}-(5 D+3) \bar{a}^{2}\right. \\
& -(D-1)\}+3(D-5) \bar{a}^{4}-6 \bar{a}^{2}+D-3 .
\end{aligned}
$$

The isentropic moment of inertia at constant pressure (analogous to $\kappa_{S}$ for a gas and evaluated in [38]) is

$$
\mathcal{I}_{S, P}=\left.\frac{\partial J}{\partial \Omega}\right|_{S, P}=\frac{r_{h} S\left(1+a^{2}\right)^{2}\left\{D-2+(D-4) \lambda \bar{a}^{2}\right\}}{2 \pi\left(1-\bar{\lambda} \bar{a}^{2}\right)^{2}\left\{D-2-(D-4) \bar{a}^{2}\right\}} .
$$

The isothermal moment of inertial tensor $\mathcal{I}_{T, P}$ can be obtained from the identity $C_{\Omega, P} \mathcal{I}_{S, P}=C_{J, P} \mathcal{I}_{T, P}$,

$$
\mathcal{I}_{T, P}=\frac{r_{h} S Z_{J}(\bar{\lambda}, \bar{a})}{2 \pi\left(1-\bar{\lambda} \bar{a}^{2}\right)^{2} Z_{\Omega}(\bar{\lambda}, \bar{a})} .
$$

The Ruppeiner metric has Ricci scalar

$\tilde{R}=-\frac{(D-3)\left(1-\bar{\lambda}^{2} \bar{a}^{4}\right) \tilde{F}_{1}(\bar{\lambda}, \bar{a}) \tilde{F}_{2}(\bar{\lambda}, \bar{a})}{\pi \varpi r_{h}^{D-1} T\left(1+\bar{a}^{2}\right)^{2}\left\{D-2+(D-4) \bar{\lambda} \bar{a}^{2}\right\} Z_{\Omega}^{2}(\bar{\lambda}, \bar{a})}$,

where $\tilde{F}_{1}(\bar{\lambda}, \bar{a})$ is linear in $\lambda$ and $\bar{a}^{2}$ while $\tilde{F}_{2}(\bar{\lambda}, \bar{a})$ is quadratic. Explicitly

$\tilde{F}_{1}(\bar{\lambda}, \bar{a})=(D-3)\left(1+\bar{\lambda} \bar{a}^{2}\right)-3\left\{(D-1) \bar{\lambda}+(D-5) \bar{a}^{2}\right\}$,

$$
\tilde{F}_{2}(\bar{\lambda}, \bar{a})=\tilde{A}_{2}(\bar{a}) \bar{\lambda}^{2}+\tilde{B}_{2}(\bar{a}) \bar{\lambda}+\tilde{C}_{2}(\bar{a}),
$$


with

$$
\begin{aligned}
\tilde{A}_{2}(\bar{a})= & (D-4)\left\{(D-3) \bar{a}^{2}-(D-1)\right\} \bar{a}^{2}, \\
\tilde{B}_{2}(\bar{a})= & (D-5)(D-6) \bar{a}^{4}-2\left(D^{2}-6 D+1\right) \bar{a}^{2} \\
& +D(D-1), \\
\tilde{C}_{2}(\bar{a})= & (D-2)\left\{(D-3)-(D-5) \bar{a}^{2}\right\} .
\end{aligned}
$$

Note that the only singularities in $R$ or $\tilde{R}$ are those associated with the spinodal curve of $C_{\Omega, P}, Z_{\Omega}=0$.

When $\bar{\lambda}=0$, Eq. (B9) reduces to

$\tilde{R}=-\frac{4(D-3)\left\{D-3-3(D-5) \bar{a}^{2}\right\}}{\varpi r_{h}^{D-2}\left(1+\bar{a}^{2}\right)\left\{(D-3)^{2}-(D-5)^{2} \bar{a}^{4}\right\}}$,

which is the result quoted in [15].
There is a critical point on the spinodal curve for $C_{J, P}$. This was first found for $D=4$ in [47], where the critical values are $\bar{\lambda}_{\text {crit }}=0.2105$ and $\bar{a}_{\text {crit }}=0.1795$, and is present in any space dimension greater than three. In five dimensions, for example, $\bar{\lambda}_{\text {crit }}=0.3569$ and $\bar{a}_{\text {crit }}=0.1802$. There is no critical point visible in $C_{\Omega, P}$; in fact $Z_{\Omega}>0$ and $C_{\Omega}<0$ at the critical point, and the critical point is actually unstable if $J$ is not fixed. The curvature scalars diverge on the spinodal curve for $C_{\Omega, P}$, not that of $C_{J, P}$, and the critical point is not visible in the thermodynamic curvature.

Allowing $P$ to vary requires using $V$ as a thermodynamic variable in order to ensure a positive definite Weinhold metric, associated with $U(S, J, V)$, in regions of the state space where the black hole is thermodynamically stable. The Weinhold metric following from (14), (28), (29), and (30) is

$$
g=\frac{\varpi r_{h}^{D-5}}{16 \pi} \times\left[\begin{array}{ccc}
\frac{\left(1+\bar{a}^{2}\right) p_{2}(\bar{\lambda}, \bar{a})}{\left(1-\bar{\lambda} \bar{a}^{2}\right)^{2}} & -\frac{2(D-2) r_{h}\left(1-\bar{\lambda}^{2}\right) \bar{a}}{\left(1-\bar{\lambda} \bar{a}^{2}\right)^{2}} & -\frac{2 r_{h}[(D-1)-(D-3) \bar{\lambda}]\left(1+\bar{a}^{2}\right) \bar{a}^{2}}{\left(1-\bar{\lambda} \bar{a}^{2}\right)^{2}} \\
-\frac{2(D-2) r_{h}\left(1-\bar{\lambda}^{2}\right) \bar{a}}{\left(1-\bar{\lambda} \bar{a}^{2}\right)^{2}} & \frac{2 r_{h}^{2}(1+\bar{\lambda})^{2}\left(\bar{a}^{2}\left(3-\bar{a}^{2}\right) \bar{\lambda}+1-3 \bar{a}^{2}\right)}{\left(1+\bar{a}^{2}\right)\left(1-\bar{\lambda} \bar{a}^{2}\right)^{3}} & \frac{r_{h}^{2}(1+\bar{\lambda})\left(\bar{\lambda} \bar{a}^{2}+1-2 \bar{a}^{2}\right) \bar{a}}{\left(1-\bar{\lambda} \bar{a}^{2}\right)^{3}} \\
-\frac{2 r_{h}[(D-1)-(D-3) \bar{\lambda}]\left(1+\bar{a}^{2}\right) \bar{a}^{2}}{\left(1-\bar{\lambda} \bar{a}^{2}\right)^{2}} & \frac{r_{h}^{2}(1+\bar{\lambda})\left(\bar{\lambda} \bar{a}^{2}+1-2 \bar{a}^{2}\right) \bar{a}}{\left(1-\bar{\lambda} \bar{a}^{2}\right)^{3}} & \frac{2 r_{h}^{2}\left(1-\bar{a}^{4}\right) \bar{a}^{2}}{\left(1-\bar{\lambda} \bar{a}^{2}\right)^{3}}
\end{array}\right],
$$

where the top left entry involves

$$
\begin{aligned}
p_{2}(\bar{\lambda}, \bar{a})= & -D(D-3) \bar{a}^{2} \bar{\lambda}^{2}+\left\{\left(D^{2}-5 D+8\right) \bar{a}^{2}\right. \\
& +(D-1)(D-2)\} \bar{\lambda}-(D-2)(D-3) .
\end{aligned}
$$

The determinant of the Weinhold metric, in $\left(r_{h}, \bar{\lambda}, \bar{a}\right)$ coordinates, is

$$
\operatorname{det} g=-\frac{\varpi^{3}(D-3) r_{h}^{3 D-11}(1+\bar{\lambda})^{3}\left(1+\bar{a}^{2}\right) \bar{a}^{4} Z_{\Omega}(\bar{\lambda}, \bar{a})}{2048 \pi^{3}\left(1-\bar{\lambda} \bar{a}^{2}\right)^{6}},
$$

which, for $\bar{\lambda}>0$, vanishes when $\bar{a}=0$ and when

$$
\bar{\lambda}:=\bar{\lambda}_{\infty}=\frac{(D-3)-(D-5) \bar{a}^{2}}{(D-1)-(D-3) \bar{a}^{2}},
$$

and diverges for

$$
\bar{\lambda} \bar{a}^{2}=1
$$

The zeros of det $g$ are genuine curvature singularities and are reflected in the Ricci scalars below; indeed these reflect singularities in the response functions: the black hole is incompressible for $\bar{a}^{2}=0$, and the heat capacity at constant angular velocity, $C_{\Omega, P}$, diverges on $\bar{\lambda}_{\infty}$ where $Z_{\Omega}=0$. Both Ricci scalars vanish at the limit of thermodynamic state space where $\bar{\lambda} \bar{a}^{2}=1$ and extensive quantities diverge.
The Ricci scalar following from the Weinhold metric (B13) can be written as a ratio of two polynomials in the dimensionless variables $\left(\bar{\lambda}, \bar{a}^{2}\right)$

$$
R=\frac{16 \pi}{(D-3) \varpi r_{h}^{D-3}} \frac{\left(1-\bar{\lambda} \bar{a}^{2}\right) F_{2}\left(\bar{\lambda}, \bar{a}^{2}\right)}{\bar{a}^{4}\left(1+\bar{a}^{2}\right)(1+\bar{\lambda})^{2} Z_{\Omega}^{2}(\bar{\lambda}, \bar{a})}
$$

where $F_{2}$ is quadratic in $\bar{\lambda}$ and quartic in $\bar{a}^{2}$. Explicitly

$$
F_{2}\left(\bar{\lambda}, \bar{a}^{2}\right)=A_{2}\left(\bar{a}^{2}\right) \bar{\lambda}^{2}+B_{2}\left(\bar{a}^{2}\right) \bar{\lambda}+C_{2}\left(\bar{a}^{2}\right)
$$

with

$$
\begin{aligned}
A_{2}\left(\bar{a}^{2}\right)= & \bar{a}^{2}\left[(D-3)(7 D-27) \bar{a}^{6}-\left(5 D^{2}-10 D-19\right) \bar{a}^{4}\right. \\
& \left.-(D-1)(3 D-13) \bar{a}^{2}+(D-1)^{2}\right], \\
B_{2}\left(\bar{a}^{2}\right)= & -(D-2)(D-5)(2 D-7) \bar{a}^{8} \\
& +\left(D^{3}-9 D^{2}+20 D-4\right) \bar{a}^{6} \\
& +\left(3 D^{3}-17 D^{2}+22 D+12\right) \bar{a}^{4} \\
& -(D-1)\left(D^{2}-8\right) \bar{a}^{2}-(D-1)^{2}(D-2), \\
C_{2}\left(\bar{a}^{2}\right)= & (D-3)(D-5)^{2} \bar{a}^{8}+(D-5)\left(D^{2}-11 D+19\right) \bar{a}^{6} \\
& -\left(3 D^{3}-28 D^{2}+70 D-61\right) \bar{a}^{4} \\
& -\left(D^{3}-12 D^{2}+30 D-23\right) \bar{a}^{2} \\
& +(D-1)(D-2)(2 D-5) .
\end{aligned}
$$


The only singularities of $R$ are those associated with the zeros of $Z_{\Omega}$.

The Ricci scalar for the Ruppeiner metric conformal to (B13) is more complicated,

$$
\tilde{R}=\frac{\left(1-\bar{\lambda} \bar{a}^{2}\right) \tilde{F}_{3}(\bar{\lambda}, \bar{a})}{4 \pi(D-3) \varpi r_{h}^{D-1} \bar{a}^{4}\left(1+\bar{a}^{2}\right)^{3}(1+\bar{\lambda})^{2} T Z_{\Omega}^{2}},
$$

where $\tilde{F}_{3}(\bar{\lambda}, \bar{a})$ is a quintic polynomial in $\bar{\lambda}$ and sixth order in $\bar{a}^{2}$. Again the only singularities are associated with the zeros of $Z_{\Omega}$.

[1] S. W. Hawking, Nature (London) 248, 30 (1974); Commun. Math. Phys. 43, 199 (1975); Phys. Rev. D 13, 191 (1976).

[2] S. W. Hawking and D. N. Page, Commun. Math. Phys. 87, 577 (1983).

[3] D. Kastor, S. Ray, and J. Traschen, Classical Quantum Gravity 26, 195011 (2009).

[4] P. Weinhold, Phys. Today 29, No. 3, 23 (1976).

[5] G. Ruppeiner, Phys. Rev. A 20, 1608 (1979).

[6] G. Ruppeiner, Rev. Mod. Phys. 67, 605 (1995).

[7] S. Ferrara, G. W. Gibbons, and R. Kallosh, Nucl. Phys. B500, 75 (1997).

[8] R. G. Cai and J. H. Cho, Phys. Rev. D 60, 067502 (1999).

[9] J. Åman, I. Bengtsson, and N. Pidokrajt, Gen. Relativ. Gravit. 35, 1733 (2003).

[10] G. Arcioni and E. Lozano-Tellechea, Phys. Rev. D 72, 104021 (2005).

[11] J. Åman and N. Pidokrajt, Phys. Rev. D 73, 024017 (2006).

[12] J. Shen, R. G. Cai, B. Wang, and R. K. Su, Int. J. Mod. Phys. A 22, 11 (2007).

[13] B. Mirza and M. Zamani-Nasab, J. High Energy Phys. 06 (2007) 059.

[14] H. Quevedo, Gen. Relativ. Gravit. 40, 971 (2008).

[15] J. Åman and N. Pidokrajt, EAS Publ. Ser. 30, 269 (2008).

[16] G. Ruppeiner, Phys. Rev. D 78, 024016 (2008).

[17] H. Quevedo and A. Sánchez, J. High Energy Phys. 09 (2008) 034.

[18] A. Sahay, T. Sarkar, and G. Sengupta, J. High Energy Phys. 07 (2010) 082.

[19] R. Banerjee, S. Ghosh, and D. Roychowdhury, Phys. Lett. B 696, 156 (2011).

[20] C. Niu, Y. Tian, and X. N. Wu, Phys. Rev. D 85, 024017 (2012).

[21] Y. D. Tsai, X. N. Wu, and Y. Yang, Phys. Rev. D 85, 044005 (2012).

[22] A. Bravetti, D. Momeni, R. Myrzakulov, and H. Quevedo, Gen. Relativ. Gravit. 45, 1603 (2013).

[23] H. Quevedo, M. N. Quevedo, A. Sanchez, and S. Taj, Phys. Scr. 89, 084007 (2014).

[24] J.-X. Mo and W.-B. Liu, Adv. High Energy Phys. 2014, 739454 (2014).

[25] G. Ruppeiner, in Breaking of Supersymmetry and Ultraviolet Divergences in Extended Supergravity, edited by
S. Bellucci, Springer Proceedings in Physics, Vol. 153 (Springer, New York, 2014), p. 179.

[26] S. A. H. Mansoori and B. Mirza, Eur. Phys. J. C 74, 2681 (2014).

[27] M. Henneaux and C. Teitelboim, Phys. Lett. 143B, 415 (1984).

[28] Y. Sekiwa, Phys. Rev. D 73, 084009 (2006).

[29] S. Wang, S. Wu, F. Xie, and L. Dan, Chin. Phys. Lett. 23, 1096 (2006).

[30] M. Urano, A. Tomimatsu, and H. Saida, Classical Quantum Gravity 26, 105010 (2009).

[31] H. Lu, Y. Pang, C. N. Pope, and J. Vazquez-Poritz, Phys. Rev. D 86, 044011 (2012).

[32] T. Padmanabhan, Phys. Rep. 380, 235 (2003).

[33] A. Larrañaga and S. Mojica, Abraham Zelmanov J. 5, 68 (2012).

[34] J. L. Zhang, R. G. Cai, and H. Yu, J. High Energy Phys. 02 (2015) 143.

[35] J. L. Zhang, R. G. Cai, and H. Yu, Phys. Rev. D 91, 044028 (2015).

[36] H. Quevedo, J. Math. Phys. (N.Y.) 48, 013506 (2007).

[37] L. Smarr, Phys. Rev. Lett. 30, 71 (1973).

[38] B. P. Dolan, Classical Quantum Gravity 31, 165011 (2014).

[39] O. J. C. Dias, P. Figueras, R. Monteiro, H. S. Reall, and J. E. Santos, J. High Energy Phys. 05 (2010) 076.

[40] G. W. Gibbons, M. J. Perry, and C. N. Pope, Classical Quantum Gravity 22, 1503 (2005).

[41] A. Chamblin, R. Emparan, C. V. Johnson, and R. C. Myers, Phys. Rev. D 60, 064018 (1999).

[42] A. Chamblin, R. Emparan, C. V. Johnson, and R. C. Myers, Phys. Rev. D 60, 104026 (1999).

[43] B. P. Dolan, Classical Quantum Gravity 28, 235017 (2011).

[44] G. W. Gibbons, H. Lü, D. N. Page, and C. N. Pope, Phys. Rev. Lett. 93, 171102 (2004).

[45] G. W. Gibbons, H. Lü, D. N. Page, and C. N. Pope, J. Geom. Phys. 53, 49 (2005).

[46] S. W. Hawking, C. J. Hunter, and M. M. Taylor-Robinson, Phys. Rev. D 59, 064005 (1999).

[47] M. M. Caldarelli, G. Cognola, and D. Klemm, Classical Quantum Gravity 17, 399 (2000).

[48] B. P. Dolan, Phys. Rev. D 84, 127503 (2011). 